This is the submitted version of the following article:

Schaefer N., Garcia-Cortadella R., Calia A.B., Mavredakis N., Illa X., Masvidal-Codina E., Cruz J.D.L., Corro E.D., Rodríguez L., Prats-Alfonso E., Bousquet J., Martínez-Aguilar J., Pérez-Marín A.P., Hébert C., Villa R., J iménez D.,

Guimerà-Brunet A., Gar. I mproved metal-graphene contacts for low-noise, high-density microtransistor arrays for neural sensing. Carbon, (2020). 161. : 647 - .

10.1016/j.carbon.2020.01.066,

which has been published in final form at https://dx.doi.org/10.1016/j.carbon.2020.01.066 () https://dx.doi.org/10.1016/j.carbon.2020.01.066. This manuscript version is made available under the CC-BY-NC-ND 4.0 license http://creativecommons.org/licenses/by-nc-nd/4.0/ 


\title{
Improved metal-graphene contacts for low-noise, high-density microtransistor arrays for neural sensing
}

\author{
Nathan Schaefer ${ }^{\mathrm{a}, \mathrm{b} \dagger}$, Ramon Garcia-Cortadella ${ }^{\mathrm{a}, \mathrm{b} \dagger}$, Andrea Bonaccini \\ Calia $^{a, b \dagger}$, Nikolaos Mavredakis ${ }^{b}$, Xavi Illa ${ }^{c, d}$, Eduard Masvidal-Codina ${ }^{c}$, \\ Jose de la Cruz ${ }^{a}$, Elena del Corro ${ }^{a}$, Laura Rodríguez ${ }^{a}$, Elisabet Prats- \\ Alfonso $^{c, d}$, Jessica Bousquet ${ }^{\mathrm{a}}$, Javier Martínez-Aguilar ${ }^{c, d}$, Antonio P. \\ Pérez-Marín ${ }^{a}$, Clement Hébert ${ }^{\mathrm{e}}$, Rosa Villa ${ }^{c, d}$, David Jiménez ${ }^{\mathrm{b}}$, Anton \\ Guimerà-Brunet ${ }^{c, d}$ and Jose A. Garrido ${ }^{\text {a,f* }}$
}

${ }^{\text {a }}$ Catalan Institute of Nanoscience and Nanotechnology (ICN2), CSIC and The Barcelona Institute of Science and Technology, Campus UAB, Bellaterra, Spain

${ }^{b}$ Departament d'Enginyeria Electrònica, Escola d'Enginyeria, Universitat Autònoma de Barcelona, Bellaterra, Spain

${ }^{\mathrm{c}}$ Instituto de Microelectrónica de Barcelona IMB-CNM (CSIC), Esfera UAB, Bellaterra, Spain

${ }^{\mathrm{d}}$ Centro de Investigación Biomédica en Red en Bioingeniería, Biomateriales y Nanomedicina (CIBERBBN), Madrid, Spain

${ }^{\mathrm{e}}$ Inserm and Université Grenoble Alpes, Saint Martin d'Hères, France

${ }^{\mathrm{f}}$ ICREA, Barcelona, Spain

$\dagger$ These authors contributed equally to this work

Received xxxxxx

Accepted for publication $\mathrm{xxxxxx}$

Published xxxxxx

\begin{abstract}
Poor metal contact interfaces are one of the main limitations preventing unhampered access to the full potential of two-dimensional materials in electronics. Here we present graphene solution-gated fieldeffect-transistors (gSGFETs) with strongly improved linearity, homogeneity and sensitivity for small sensor sizes, resulting from ultraviolet ozone (UVO) contact treatment. The contribution of channel and contact region to the total device conductivity and flicker noise is explored experimentally and explained with a theoretical model. Ultimately, in-vitro recordings of flexible microelectrocorticography ( $\mu$-ECoG) probes were performed to validate the superior sensitivity of the UVO-treated gSGFET to brain-like activity. These results connote an important step towards the fabrication of high-density gSGFET $\mu$-ECoG arrays with state-of-the-art sensitivity and homogeneity, thus demonstrating the potential of this technology as a versatile platform for the new generation of neural interfaces.
\end{abstract}

\footnotetext{
*Corresponding author. Tel: +34937374648. E-mail: joseantonio.garrido@icn2.cat (Jose Antonio Garrido)
} 


\section{Introduction}

With its exceptional properties such as high mobility [1], biocompatibility [2], transparency [3] and mechanical strength [4], graphene has been extensively investigated for application in numerous fields such as electonics [5], photonics and optoelectronics [6], and more recently biomedical engineering [7][9]. Despite the vast potential of graphene, graphene-based devices often fall short of demonstrating their full capabilities, as large metal-graphene contact resistance [10], inhomogeneity among devices [11] and high 1/f noise [12] still hamper their performance. Hence, great efforts have been undertaken to properly understand the source of noise and of the high contact resistance as well as to find ways to minimize their impact on the device performance.

In a graphene-based field-effect transistor (FET), we can distinguish several contibutions to low-frequency noise [12]. For instance, variations in the scattering cross-section of lattice defects generate mobility fluctuations which ultimately cause changes in the resistance of graphene. In addition, charge trap states in the graphene channel or in the surrounding materials such as the substrate, gate dielectrics or encapsulation layers can cause fluctuations in the number of charge carriers. Due to the 2-dimensional nature of graphene, the latter is expected to be dominant, as shown for certain transistor configurations [13]. The use of buffer layer materials such as hexagonal boron nitride $(\mathrm{hBN})$ [14] or engineering free standing graphene channels [15] has been proposed to decouple the graphene sheet from the environment, and thus reduce flicker noise.

High contact resistance, resulting from the work function mismatch between graphene and metals as well as from the low density of states of graphene, has a negative impact on the performance of graphene FETs, resulting in a poor charge transfer and thus in a lower conductance of graphene FETs [10], [16], [17]. Common strategies to reduce contact resistance include the use of metals which interact strongly with graphene [18]-[20] or the creation of defects in the graphene lattice; for instance, the introduction of dangling bonds can enhance the graphene-metal interaction. Typical ways to create defects in graphene include, for instance, patterning the graphene sheet beneath the metal contacts, increasing the graphene edge length [21]-[23], as well as treating the contacted graphene with oxygen plasma [24] or ozone [25]. Despite all available reported techniques used to lower contact resistance, the goal of most published studies focuses on setting new performance benchmarks for a single device by using nanofabrication techniques and exfoliated graphene [14], [26], and only very few of them address the technique's scalability for wafer-size fabrication [27]. Since the success of 2D materials will ultimately depend on their translation from the laboratory to the industry, demonstration of large-scale fabrication processes 
with high homogeneity and high yield are of critical importance. Next to its impact on the current-voltage characteristics of the transistor, metal-graphene contacts have also been reported to exhibit time-dependent resistance fluctuations [28], contributing to the low-frequency noise. Although significant efforts have been devoted to better understand electrical noise in graphene [12], no consensus has been reached yet on whether channel or contact noise is dominating in graphene FETs, what the underlying mechanisms generating noise are and how noise depends on channel and contact geometry [26], [29]-[33].

Here we use wafer-processed graphene solution-gated field-effect transistors (g-SGFETs) to demonstrate the effect of the contact resistance on the sensing performance of these devices. gSGFETs have been widely explored for application in microelectrocorticography ( $\mu$-ECoG) arrays to record neural activity [9], [34], [35]. As neural signals are usually extremely small in amplitude (typically below $100 \mu \mathrm{V}$ ), having devices with good signal-to-noise ratio (SNR) is of paramount importance for high-quality recordings. Previous work investigated the bias dependence of low-frequency noise in gSGFETs and identified that carrier density fluctuations can generate low-frequency noise in the channel [13]. However, the impact of contact noise in g-SGFETs has not been addressed yet. In this work, in addition to present a fundamental noise study, we also explore the impact of low contact resistance on the actual recording quality of neural sensor-arrays considering factors such as signal-to-noise ratio, sensor homogeneity and linearity of signal transduction.

\section{Experimental}

\subsection{Device fabrication}

The devices for the transfer length method (TLM) study were fabricated by contact photolithography on 4' $\mathrm{Si} / \mathrm{SiO} 2$ wafers. In a first step, graphene grown by chemical vapour deposition (CVD) was transferred [34], patterned (HiPR6512 photresist, FujiFilm) and etched by deep reactive-ion etching (DRIE) for $1 \mathrm{~min}$ at $150 \mathrm{~W}$ in oxygen plasma. Subsequently, the photoresist for the contact metal layer was deposited, illuminated through a chromium mask and developed. Before evaporating 20nm Ni and 200nm Au (ebeam evaporator) the wafer was exposed to UV-ozone [Jelight Model 42] [25]. After lift-off a $2 \mu \mathrm{m}$ passivation layer is deposited (SU8-2005 MicroChem) with open windows in the channel region.

The flexible ECoG-probes were processed following the same protocol; however a $7 \mu \mathrm{m}$ layer of polyimide (PI-2611 HD MicroSystems) was spun onto the Si wafer prior to the fabrication to serve as the flexible substrate and structured via DRIE to allow individual peeling of each probe after all steps are finished. Due to the higher mechanical stress in flexible probes, an additional metal layer $(20 \mathrm{~nm} \mathrm{Ti} / 200 \mathrm{~nm} \mathrm{Au})$ 
was used to improve the adhesion between the top metal layer and the substrate, yet not directly contacting the graphene channel.

\subsection{Device characterization and recording setup}

Transfer characteristics and noise were measured with custom-built electronics, performing current-tovoltage conversion, filtering and bias-control of up to 16 transistors simultaneously. To allow sensitivity towards large DC currents and high gain for noise detection, the converted signals are split into DC (frequency $<0.1 \mathrm{~Hz})$ and $\mathrm{AC}(0.1 \mathrm{~Hz}<$ frequency $<5 \mathrm{kHz})$ components. A commercial data acquisition system (National Instruments USB-6363) was used to provide the applied bias and record the voltage signals after conversion [34].

\subsection{Raman spectroscopy and atomic force microscopy}

The Raman spectra were acquired with a Witec spectrometer in backscattering configuration, using a 600 $\mathrm{gr} / \mathrm{nm}$ grating which provided a spectral resolution of $3 \mathrm{~cm}^{-1} /$ pixel. A $488 \mathrm{~nm}$ excitation laser $(1 \mathrm{~mW}$ power) was focused on the sample with a 50x objective leading to a spatial resolution below $0.8 \mu \mathrm{m}$. The integrated peak area ratio $\left(\mathrm{A}_{\mathrm{D}} / \mathrm{A}_{\mathrm{G}}\right)$ is calculated by fitting each peak with a lorentzian function after previous background substration. Each data point shown in figure 1a was obtained from the statistical analysis of Raman mappings of $15 \times 40 \mu \mathrm{m}^{2}$ contact region. An Asylum MFP-3D atomic force microscope (AFM) was used to characterize the surface in standard air-tapping mode (figure 1c).

\subsection{Contact resistance from transfer length method}

The contact resistance $\left(\boldsymbol{R}_{\boldsymbol{c}}\right)$ and the sheet resistance $\left(\boldsymbol{R}_{\text {sheet }}\right)$ values shown in figure 1e-g have been extracted by applying the TLM to different devices of $2.5 \mu \mathrm{m}, 5 \mu \mathrm{m}, 8 \mu \mathrm{m}, 10 \mu \mathrm{m}, 20 \mu \mathrm{m}$ and $40 \mu \mathrm{m}$ channel length and $40 \mu \mathrm{m}$ channel width. For each length, the resistance value has been extracted from the mean value of several measurements and fitted with a linear regression model to extract the values for $\boldsymbol{R}_{\boldsymbol{c}}$ and $\boldsymbol{R}_{\text {sheet }}$. To further improve the accuracy of this approach, outlier resistance values which strongly deviate from the median of each transistor type (outside the $25^{\text {th }}$ and $75^{\text {th }}$ percentile) were removed.

\section{Results and discussion}

\subsection{Low contact resistance by surface cleaning and defect creation}

Ultraviolet ozone (UVO) treatment has been previously reported as an efficient way to reduce contact resistance in graphene FETs [25], [27]. Part of the effect is attributed to cleaning of fabrication residues 
which leads to a decrease of the intensity ratio of the D-peak to the G-peak $\left(A_{D} / A_{G}\right)$. However, longer treatment durations are known to break up the $\mathrm{sp}^{2}$-bonds and to create vacancies in the graphene sheet [25], which increases $\mathrm{AD} / \mathrm{Ag}_{\mathrm{g}}$. Since the $\mathrm{sp}^{2}$ orbitals hinder charge injection along the z-plane [36], [37], the creation of vacancies by the longer UVO-treatment further decrease the contact resistance. Figure 1d shows the evolution of $\mathrm{AD} / \mathrm{Ag}_{\mathrm{G}}$ as a function of treatment duration. The results are comparable to the ones presented in literature [25], with the exact duration depending on the experimental conditions of the UVOtreatment.

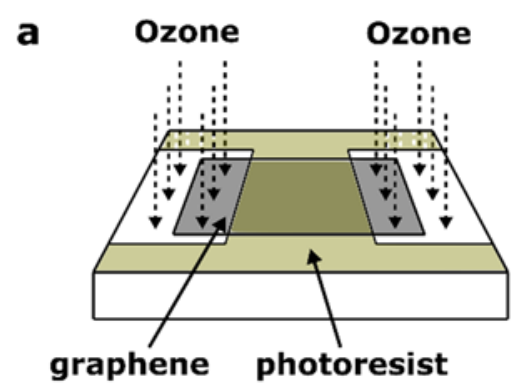

\section{C}
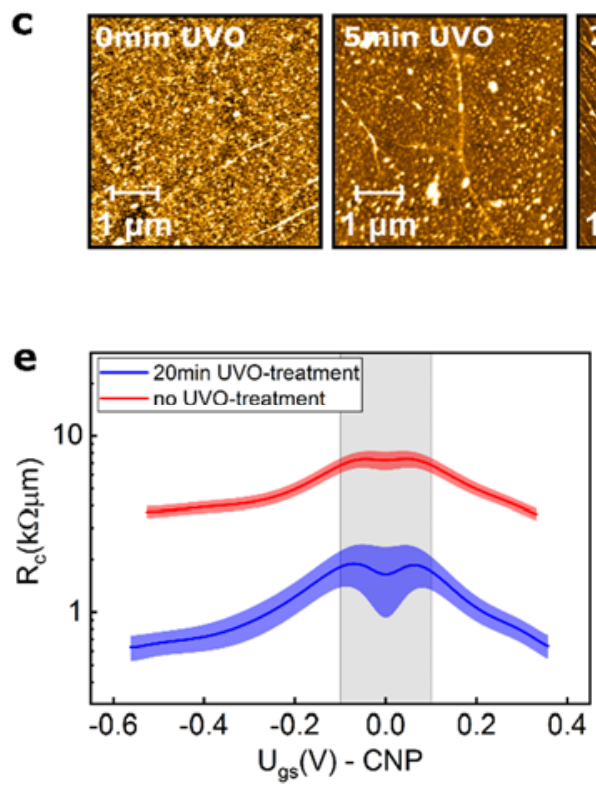
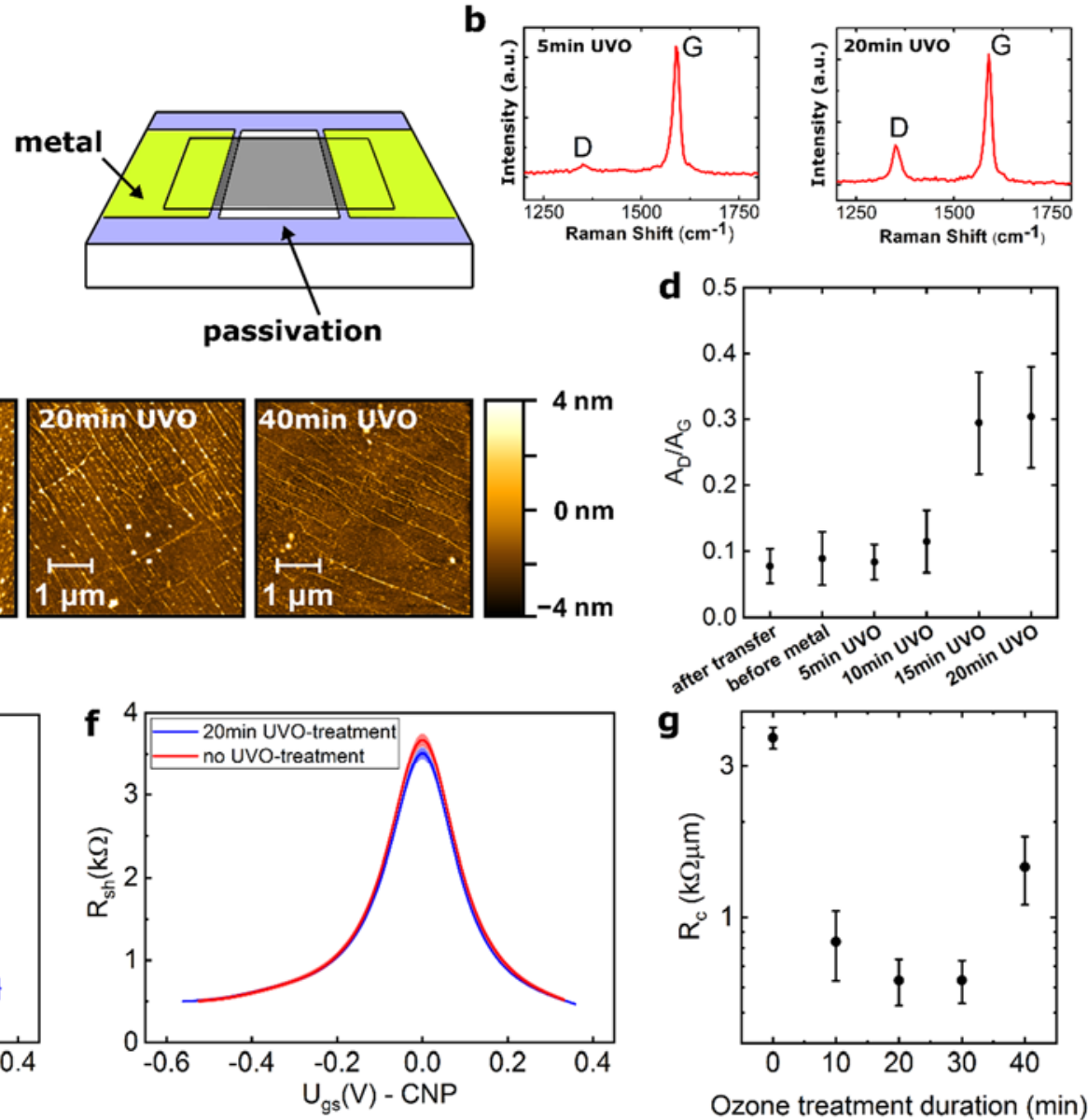

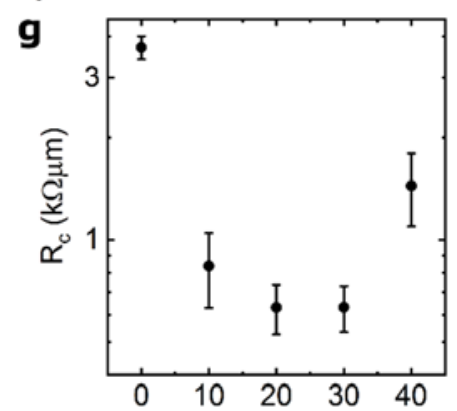

Ozone treatment duration $(\mathrm{min})$

Figure 1: Improving contact resistance by surface cleaning and defect creation. a. Schematic of device fabrication with ozone applied to the graphene contact region prior to the metal deposition. b. Raman spectra after $5 \mathrm{~min}$ and $20 \mathrm{~min}$ treatment, indicating an increase of the D-peak with treatment duration. c. Surface roughness measured by AFM indicating the continuous cleaning of surface residues. d. Ratio of peak intensities of the D-band and G-band of single-layer graphene used as an indicator for the amount of residues and defects in the graphene sheet. $\boldsymbol{R}_{\boldsymbol{c}}(\mathbf{e})$ and $\boldsymbol{R}_{\boldsymbol{s} \boldsymbol{h}}(\mathbf{f})$ measured as a function of applied gate bias for UVO-treated and non-treated devices. The shaded region close to the CNP does not yield reliable values for $\boldsymbol{R}_{\boldsymbol{c}}$, as here the applied gate bias reaches similar values as the drain-source bias $\left(\boldsymbol{U}_{\boldsymbol{d} s}=20 \mathrm{mV}\right)$, leading to a non-constant $\boldsymbol{R}_{\boldsymbol{s h}}$ along the channel. g. $\boldsymbol{R}_{\boldsymbol{c}}$ at $\mathrm{U}_{\mathrm{gs}}-\mathrm{CNP}=-0.5 \mathrm{~V}$ as a function of UVO-treatment duration, showing optimal results for $20 \mathrm{~min}$ to $30 \mathrm{~min} \mathrm{UV}$ ozone exposure. 
The change in surface topology with the UVO-treatment was analyzed by AFM. Figure 1c illustrates the surface roughness before and after treatments of varying durations, showing continuous cleaning of residues with longer treatments, which leads to a lowering of the measured roughness and a better visualization of the wrinkled surface of the graphene sheet. Figure 1e-f show the influence of the treatment on the contact resistance and sheet resistance of the graphene channel. The symmetric decrease (for electrons and holes) of $R_{c}$ away from the charge neutrality point (CNP) has been previously reported for back-gated graphene FETs using Ni contacts [16], [19] and has been associated to the poor charge screening in 2D materials with low charge carrier densities [38]. It is important to note that the TLM assumes a constant sheet resistance per unit area across the whole transistor channel. However, this assumption is only correct for cases in which the applied gate bias is much larger than the drain-source bias. While this is mostly true for field-effect-transistors based on relatively thick dielectrics, solutiongated FETs are normally operated at much lower gate voltages. In such case, the potential difference between channel and gate is not constant but changes gradually along the channel, which leads to a nonuniform sheet resistance and can result in unreliable values for $\boldsymbol{R}_{\boldsymbol{c}}$ in the grey shaded region of figure $1 \mathrm{e}$. Extracted values for $\boldsymbol{R}_{\boldsymbol{c}}$ far away from the CNP (figure 1g), yield around 3-4 $\mathrm{k} \Omega \mu \mathrm{m}$ for the case of a pristine $\mathrm{Ni} / \mathrm{Au}$ top-contact structure and reaches a minimum as low as $600 \Omega \mu \mathrm{m}$ for a $20-30 \mathrm{~min}$ UVOtreatment duration. Longer treatments result in an increase of contact resistance, as a high defect density in the UVO-treated graphene sheet eventually hamper charge conduction. On the other hand, the sheet resistance remains independent of the UVO-treatment as expected, since the channel region is protected by the photoresist.

\subsection{Improved linearity of signal transduction, homogeneity and signal-to-noise ratio in short channel transistors}

When planning the use of gSGFETs as a transducer for neural activity, there are several factors to be considered to obtain distortion-free, high-resolution recordings. Figure 2 illustrates how contact resistance influences the sensing performance of gSGFETs, by comparing devices (of varying channel length and 40 $\mu \mathrm{m}$ channel width) with low (20min UVO-treatment) and high contact resistance (no UVO-treatment). The suitability of the gSGFET for sensing applications, depends on its linear transfer characteristics, converting the voltage variation of a signal on the gate into a current modulation between the drain and source terminal. A high contact resistance at the metal graphene interface causes a flattening of the transfer curve away from the CNP (figure 2a), limiting the range of linear operation. The resulting non-linearities can cause distortions in the transduced signal, thus degrading the recording quality of the graphene 
transistors. Furthermore, a flattening of the transfer curve also limits the gSGFET's transconductance, defined as the change of drain-source current induced by a changing gate bias $\left(G_{m}=d I_{d s} / d U_{g s}\right)$, which is a figure of merit for the device's sensitivity. The gSGFET is commonly operated at the bias conditions which provide peak-transconductance (illustrated in figure $2 b$ ). Figure $2 \mathrm{~d}$ shows how the normalized peaktransconductance value changes for devices of varying channel length $(\mathrm{L}=5 \mu \mathrm{m}, 8 \mu \mathrm{m}, 10 \mu \mathrm{m}, 20 \mu \mathrm{m}$, $40 \mu \mathrm{m}, 60 \mu \mathrm{m})$ and constant channel width $(\mathrm{W}=40 \mu \mathrm{m})$. In case of low $R_{c}$ (i.e. for UVO-treated devices) the $G_{m} / U_{d s}$ exhibits a near linear increase with $\mathrm{W} / \mathrm{L}$, while for devices with high $R_{c}$ (non-treated) it is increasingly limited for short channels. As high transconductance is generally desirable for sensing applications to allow for best signal transduction, the influence of the contact resistance has to be considered for the choice of channel geometry of the sensor.

a

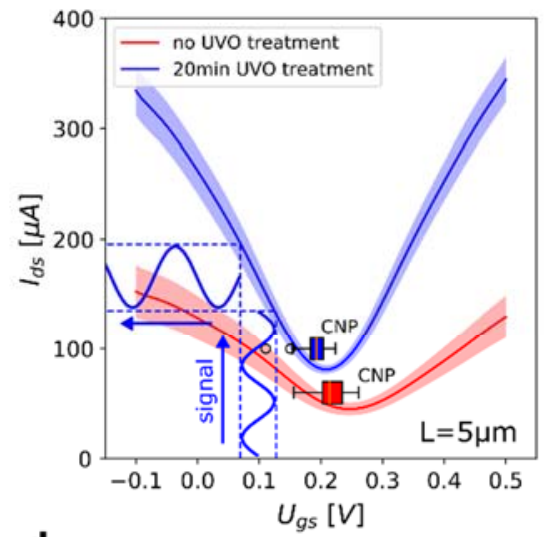

d

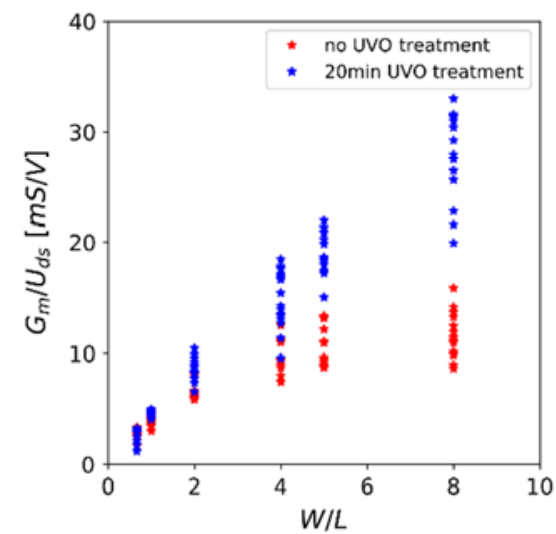

b

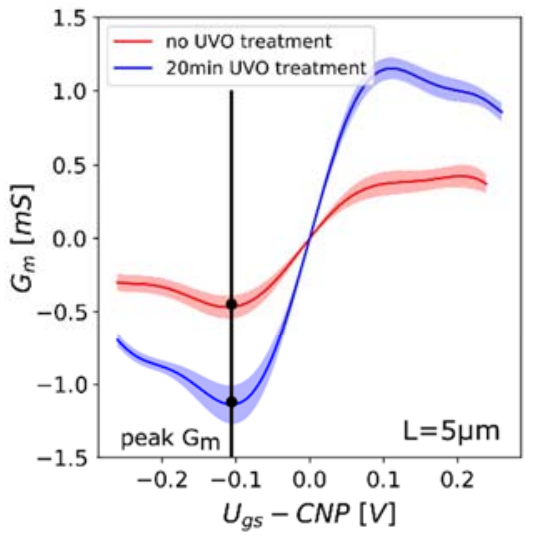

e

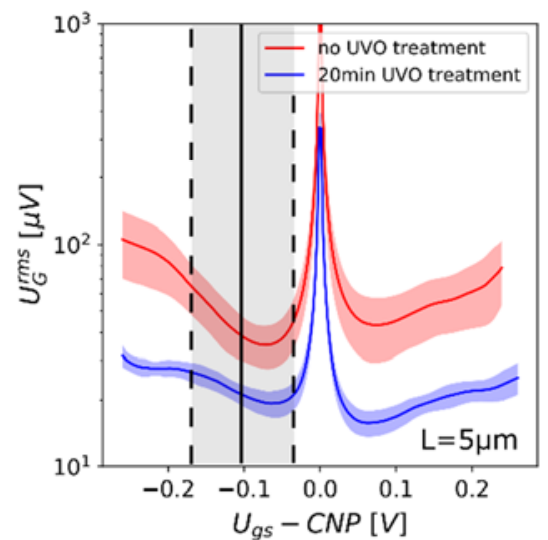

C

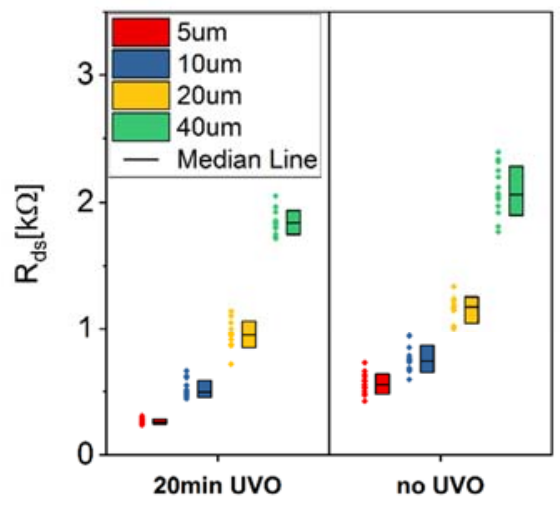

f

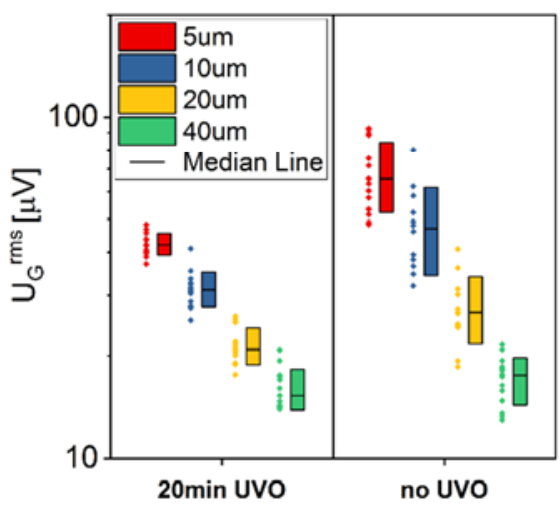

Figure 2: Effect of contact resistance on linearity of signal transduction, homogeneity and signal-to-noise ratio of gSFGETs. Average value and standard deviation of transfer curves for $\mathbf{a} . \boldsymbol{I}_{\boldsymbol{d} \boldsymbol{s}}, \mathbf{b} . \boldsymbol{G}_{\boldsymbol{m}}$ and $\mathbf{e} . \mathbf{U}_{\mathbf{G}}{ }^{\text {rms }}$ for devices of $5 \mu \mathrm{m}$ channel length and $40 \mu \mathrm{m}$ channel width. $d$. Normalized transconductance $\mathrm{G}_{\mathrm{m}} / \mathrm{U}_{\mathrm{ds}}$ (values within $25^{\text {th }}$ and $75^{\text {th }}$ percentile) versus widthto-length ratio of the channel. The statistical variation of the $\boldsymbol{R}_{\boldsymbol{d} \boldsymbol{s}}$ (c) and $\mathbf{U}_{\mathbf{G}}{ }^{\text {rms }}$ (f) values at peak transconductance $\left(\boldsymbol{U}_{\boldsymbol{g s}}=\mathrm{CNP}-\right.$ $0,1 \mathrm{~V})$ for devices of different length are also shown. 
Arguably, one of the most critical performance indicator of the gSGFET is its equivalent rms gate noise $U_{G}{ }^{r m s}$ (figure 2e), which relates the intrinsic rms current noise $I_{d s}^{r m s}$ (integrated current noise over the frequency bandwidth of interest; here filtered between $1-100 \mathrm{~Hz}$ ) of the transistor with its transconductance $\left(U_{G}{ }^{r m s}=I_{d s}{ }^{r m s} / G_{m}\right)$, and represents the detection theshold for signals applied to the gate. At peak- $G_{m}$ a significant reduction of the effective gate noise can be seen in the UVO-treated devices, which is most striking for short length. This difference becomes more pronounced when moving further away from the CNP: $U_{G}{ }^{r m s}$ remains fairly constant in the case of low $R_{C}$ and increases rapidly for devices with high $R_{c}$. This is particularly interesting when operating the gSGFET in-vivo, which typically requires to use a common bias point for all devices. However, local changes in doping across the different recording sites, as well as electrochemical drifts of the reference electrode during chronic recordings make it virtually impossible to operate all devices simultaneously at their ideal bias condition. As neural interfaces are mainly considered for long-term chronic recordings, ranging from several days up to months or even years of implantation, stable recording quality is mandatory. Consequently, having a constant SNR across a large bias window, resulting from a voltage-independent $U_{G}^{r m s}$, is crucial to provide optimal sensing capabilities across the sensor array throughout long recording times. In addition to the positive effect of the UVO-treatment on the contact resistance and noise of the devices, figure $2 \mathrm{f}$ shows that the corresponding dispersions across different recording sites are significantly reduced in the case of devices with the UVO-treatment. This suggests that poor contact interfaces are responsible for the dispersion observed in the array in terms of noise (figure $2 \mathrm{f}$ ) and conductance (figure $2 \mathrm{c}$ ), possibly overruling the contribution of the dispersion in the graphene quality in the channel. High homogeneity across recording sites is indispensable for any sort of sensor array, as it allows uniform recording quality.

\subsection{Noise contributions from contact and channel}

The improvement in the device performance resulting from the contact treatment directly leads to two questions: i) what is the contribution from the contact noise with respect to the channel noise?, and ii) what is the reason for the contact noise improvement?. Answering these questions is critical to know to which extend contact noise mitigation will affect the total noise in g-SGFETs, and to understand how to optimize the contact treatment procedure. The total normalized noise $\boldsymbol{S}_{\boldsymbol{I}} / \boldsymbol{I}_{\boldsymbol{d}}{ }^{2}$ generated in a gSGFET includes the contribution from the contacts $\left(\boldsymbol{S}_{\boldsymbol{R}_{\boldsymbol{c}}}\right)$ and from the channel $\left(\boldsymbol{S}_{\boldsymbol{R}_{\boldsymbol{c}}}\right)$ (see figure 3a-top). The contribution of these terms to the measured current noise can thus be added linearly with the proper normalization: 


$$
\frac{S_{I}}{I_{d s}^{2}}=\frac{S_{R_{c}}+S_{R_{c h}}}{R_{T}^{2}}
$$

Eq. 1

where $\boldsymbol{R}_{\boldsymbol{T}}$ is the total resistance of the transistor. The dependence of each of these terms on the channel width $(\boldsymbol{W})$ and length $(\boldsymbol{L})$ will determine their relative contribution to the total noise for different geometries and sizes. The geometrical dependence of $\boldsymbol{S}_{\boldsymbol{R}_{\boldsymbol{c}}}$ and $\boldsymbol{S}_{\boldsymbol{R}_{\boldsymbol{c}}}$ can be expressed explicitely:

$$
\frac{S_{I} f}{I_{d s} 4}=\left[k_{R_{c}} / W^{3}+k_{R_{c h}} L /\left(W^{3}\right)\right] / V_{d s}^{2}
$$

Eq. 2

where $k_{R_{C}}$ and $k_{R_{c h}}$ include all the constants which are independent of the channel dimensions for $S_{R_{C}}$ and $S_{R_{c h}}$ respectively (see supplementary information $\mathrm{S} 1$ ). The relative contribution of each term can therefore be identified by evaluating the noise dependence on $L$. Figure $3 \mathrm{~b}$ shows the normalized integrated current noise, $I_{d s}^{r m s} / I_{d s}{ }^{2}$ (where $I_{d s}^{r m s} \propto \sqrt{S_{I}}$ ), for different channel lengths. As demonstrated above, the measured noise is reduced for devices with the UVO-treatment. Interestingly, the derived dependence of the measured noise on $L$ indicates the transition from a regime dominated by contact noise to a regime dominated by channel noise. The channel length for which the two terms contribute equally is approximately $60 \mu \mathrm{m}$. For devices with shorter channel length the UVO-treatment results in a significant improvement on the total noise of the g-SGFETs. It is important to note that the improvement in $U_{G}{ }^{r m s}$ is not only caused by the increase in $G_{m}$ (figure 2), but also by the reduction of the intrinsic noise of the contact $S_{R_{c}}$. The transition from a contact noise to a channel noise dominated regime can be confirmed by modelling the $U_{g s}$ dependence of the measured current noise. Following the model presented in [13] that describes the $U_{g s}$ dependence of $I_{d s}{ }^{r m s}$ when channel noise dominates, and including the contribution of the contact noise using Eq. 1 (assuming a constant $R_{C}$ ), we have fitted the experimental data to separate the contributions of contact and channel to the total noise. Figure $3 \mathrm{c}$ compares the measured $I_{d s}{ }^{r m s}-U_{g s}$ for g-SGFETs with UVO-treated and non-treated contacts. In the case of the non-treated devices the contribution from the contacts dominates the total transistor noise in most of the $U_{g s}$ range. Conversely, for UVO-treated devices the $U_{g s}$ dependence of the channel noise presents a characteristic M-shape , which has been attributed to the dominance of channel noise [13]. Note that in figure $3 \mathrm{c}$ the $I_{d s}{ }^{r m s}$ is not normalized, therefore it does not directly represent the noise sources $S_{R_{c}}$ and $S_{R_{c h}}$. 

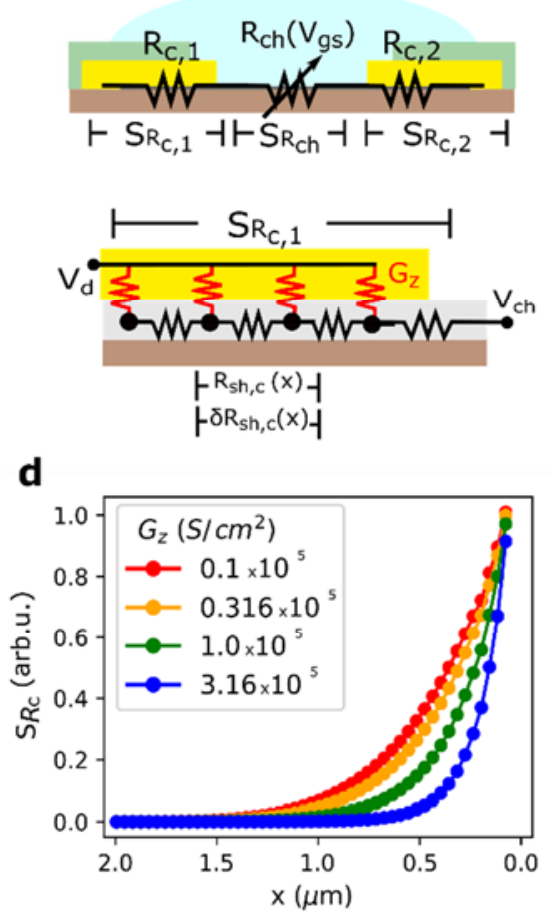
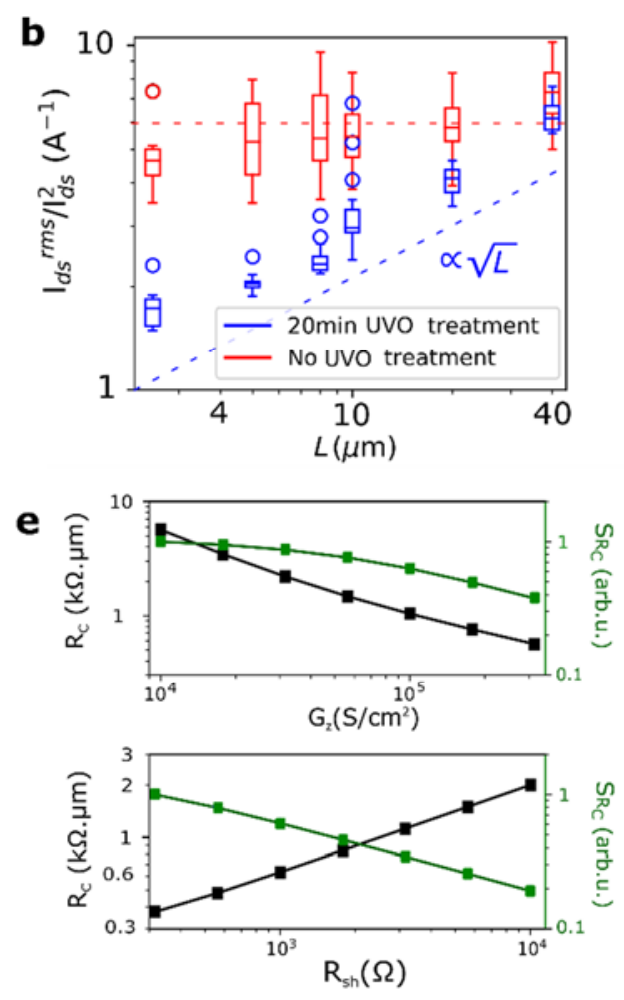

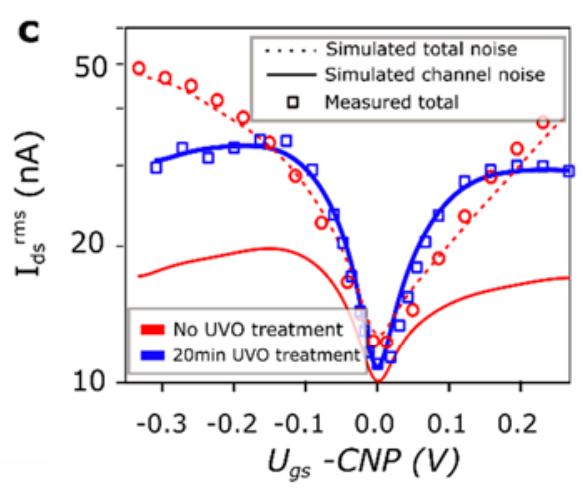

$\mathbf{f}$

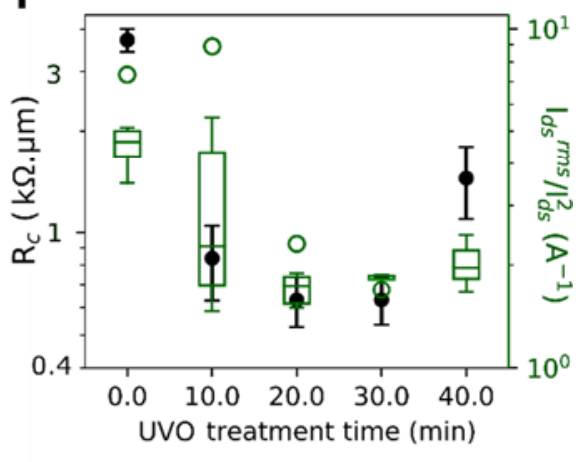

Figure 3: Geometrical dependence of contact and channel noise in gSGFETs. a. Top: schematic of a gSGFET showing the contact resistance $\left(\boldsymbol{R}_{\boldsymbol{c}}=\boldsymbol{R}_{\boldsymbol{c}, \mathbf{1}}+\boldsymbol{R}_{\boldsymbol{c}, \mathbf{2}}\right)$, channel resistance $\left(\boldsymbol{R}_{\boldsymbol{c h}}\right)$ and the noise contributions from the contacts $\left(\boldsymbol{S}_{\boldsymbol{R}_{c}}=\boldsymbol{S}_{\boldsymbol{R}_{\boldsymbol{c}, \mathbf{1}}}+\right.$ $\left.\boldsymbol{S}_{\boldsymbol{R}_{c, 2}}\right)$ and channel $\left(\boldsymbol{S}_{\boldsymbol{R}_{c h}}\right)$. Bottom: Schematic of the equivalent circuit of the transmission-line contact at the graphene-metal interface. The distributed elements representing the sheet resistance of graphene along the contact $\left(\boldsymbol{R}_{\boldsymbol{s h}, \boldsymbol{c}}(\mathrm{x})\right)$ are defined together with the local fluctuations in the sheet resistance $\left(\boldsymbol{\delta} \boldsymbol{R}_{\boldsymbol{s} \boldsymbol{h}}(\mathrm{x})\right)$. The drain voltage $\left(\boldsymbol{V}_{\boldsymbol{d}}\right)$ and the potential at the channel/contact interface $\left(\boldsymbol{V}_{\boldsymbol{c h}}\right)$ are also defined. b. Dependence of the normalized noise $\left(\boldsymbol{I}_{\boldsymbol{d s}}{ }^{r \boldsymbol{m} \boldsymbol{s}} / \boldsymbol{I}_{\boldsymbol{d s}}{ }^{2}\right)$ versus the channel length $(\boldsymbol{L})$ for devices with non-treated contacts and for devices with contacts treated with UVO for $20 \mathrm{~min}\left(\boldsymbol{U}_{\boldsymbol{g s}}=\boldsymbol{C N} \boldsymbol{P}-\right.$ $\mathbf{0 . 1 V}$ ). A change from an approximately $L$ independent regime to a $\propto \sqrt{\boldsymbol{L}}$ regime is observed. c. Experimentally obtained integrated noise $\boldsymbol{I}_{\boldsymbol{d s}}{ }^{r m s}$ versus $\boldsymbol{U}_{\boldsymbol{g} \boldsymbol{s}}$, plotted for non-treated contacts and contacts treated with UVO for $20 \mathrm{~min}$ and channel length of $5 \mu \mathrm{m}$. The lines correspond to the fitting of the experimental data with a model that considers the contributions of the channel noise (solid line) only and of the channel plus contact noise (dashed line). In UVO-treated devices the channel noise model fully descibes the $\boldsymbol{U}_{\boldsymbol{g s}}$ dependence of $\boldsymbol{I}_{\boldsymbol{d} \boldsymbol{s}}{ }^{r \boldsymbol{m} \boldsymbol{s}}$ (both curves excatly overlap). d. Local contribution of resistance fluctuations to $\boldsymbol{S}_{\boldsymbol{R}_{\boldsymbol{c}}}$ along the contact, shown for different values of conductance across graphene-metal interface $\left(\boldsymbol{G}_{\boldsymbol{z}}\right)$, according to the FEM model described in the main text. e. Evolution of $\boldsymbol{R}_{\boldsymbol{c}}$ (left axis) and $\boldsymbol{S}_{\boldsymbol{R}_{\boldsymbol{c}}}$ (right axis) with $\boldsymbol{G}_{\boldsymbol{z}}$ (top) and $\boldsymbol{R}_{\boldsymbol{s h}, \boldsymbol{c}}$ (bottom). f. Experimentally obtained $\boldsymbol{R}_{c}$ (left axis) and $\boldsymbol{I}_{d s}{ }^{r m s} / \boldsymbol{I}_{d s}{ }^{2}$ (right axis) shown for different UVO-treatment durations and channel length of $5 \mu \mathrm{m}$.

We turn now to discuss the cause of the contact noise improvement by the UVO-treatment. To this end, we start by modelling the contact in graphene with the transmission-line model equivalent circuit shown in figure 3a, with $G_{z}$ representing the conductance through the metal-graphene interface z-plane and $R_{s h, c}(x)$ representing the local values of the graphene sheet resistance at the contact. In this type of contacts, the current crowding [39] effect causes a non-homogenous current injection into the graphene sheet, with the highest current flowing next to the metal edge and the lowest at the end of the contact (see supplementary information S2). This attenuation of the current density at the contact is characterized by 
the transfer length $L_{T}$, at which the current density has decreased by a factor $e$. We suggest that the UVOtreatment causes an increase in $G_{z}$, possibly due to elimination of fabrication residues and/or the creation of defects in graphene [25]. Such defects also produce an increase in the sheet resistance of graphene $\left(R_{s h, c}\right)$ at the contacts, see figure $3 \mathrm{a}$ and [40]. Note that both the increase of $G_{z}$ and increase of $R_{s h}$ promote the current crowding effect, producing the shortening of $L_{T}$ (see supplementary information S2). Following previous work [14], we assume that the noise originates from resistance fluctuations in the graphene sheet under the metal contacts. These fluctuations can be caused by mobility fluctuations or by changes in the number of charge carriers trapped in the graphene substrate. Considering the equivalent circuit shown in Figure 3a, the power of local fluctuations in the sheet resistance of graphene $\delta R_{s h, c}$ adds up linearly. However, in this distributed elements circuit the resistance fluctuations at different positions along the contact contribute differently to the overall contact noise. The contribution of each local noise source can be calculated by weighting its noise power with the term $d R_{C} / d R_{s h, c}(\mathrm{x})$ and integrating over the whole contact length $\left(L_{c}\right)$ (see supplementary information S3). To perform this calculation, we have modelled the transmission-line contact using finite elements method (FEM), as detailed in supplementary information. Figure $3 \mathrm{~d}$ shows the calculated local contribution from each section of the contact for different values of conductance across the z-plane $\left(G_{z}\right)$. When the current crowding effect is enhanced, the contribution to noise from local sources is confined closer to the metal edge. Similarly, when $R_{s h, c}$ is increased the same effect occurs (see supplementary information S2). The total integrated contact noise $S_{R_{C}}$ is shown in figure $3 \mathrm{e}$ together with $R_{c}$ as a function of $G_{z}$ and $R_{s h, c}$, revealing that the simultaneous drop in $R_{c}$ and in $S_{R_{c}}$ can only be caused by an increase in $G_{z}$. Figure $3 \mathrm{f}$ shows the measured $R_{c}$ and $S_{R_{c}}$ of the gSGFETs for different UVO-treatment durations. It is possible to observe a monotonic decrease of both parameters until 20 min treatment duration, indicating that the contact noise mitigation in this range of UVO-treatment is caused by an increase in $G_{z}$, rather than by an increase in $R_{s h, c}$. For long treatments above $30 \mathrm{~min}$, however, the trend changes: $R_{c}$ increases with the treatment duration, while $S_{R_{c}}$ remains approximately constant (see supplementary information $\mathrm{S} 4$ ). We tentatively explain the increase in $R_{c}$ as a result of the creation of defects on the graphene sheet at the contact, that causes a significant increase in $R_{s h, c}$. These results demonstrate the reduction of the noise contributions from the contacts by UVOtreatment and offer an explanation to understand the origin and limits of this improvement. 


\subsection{Enhanced performance of flexible gSGFET ECoG-array for neural interfaces}

After the above discussion on the effect of contact resistance on the overall gSGFET performance and its low-frequency noise, we examine now how this improved performance is translated to the final application of flexible gSGFET probes for neural activity recordings. Figure $4 \mathrm{a}$ shows an illustration of this technology, combining several metal, and passivation layers on a flexible polyimide substrate (fabrication described in the experimental section), corresponding to an array of 16 microtransistors. A squared channel geometry of $20 \mu \mathrm{mx} 20 \mu \mathrm{m}$ size is used to validate the performance of the flexible probe. The distance between transistors is $400 \mu \mathrm{m}$. To exclude the variability due to the use of different graphene samples, the graphene transfer procedure or local changes of electronic properties in the graphene sheet, UVO-treated and non-treated gSGFETs are distributed in a chessboard arrangement on the same array.
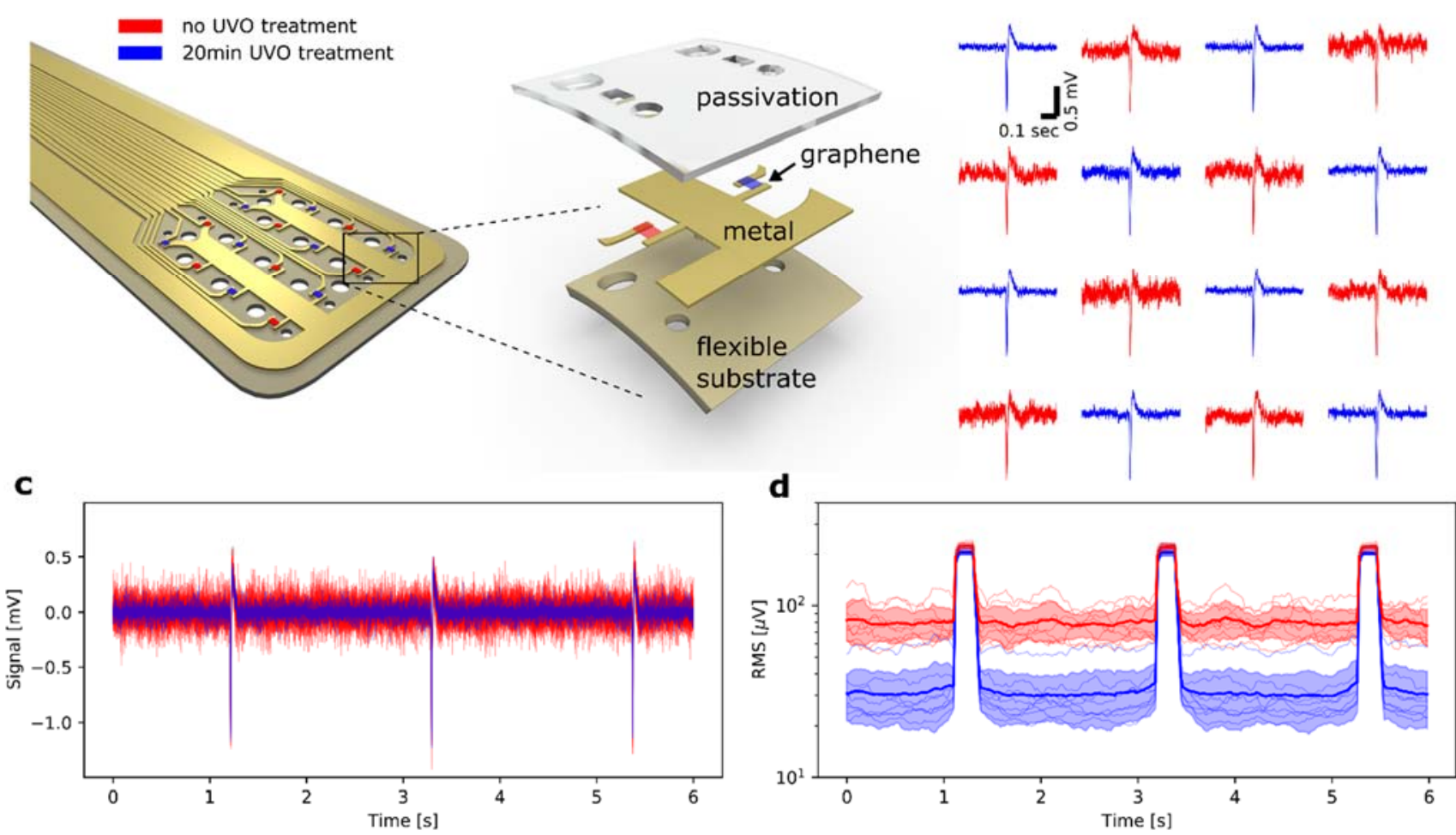

Figure 4: In-vitro assessment of recording quality of gSGFETs using hippocampal population spike-like signals. a. Schematic of a flexible epicortical probe with 16 gSGFET sensors $(20 \mu \mathrm{mx} 20 \mu \mathrm{m}$ channel area), with UVO-treated and nontreated sensors arranged in a chessboard layout. b. Map of a single spike event recorded by each transistor of the array. c. Overlapped presentation of all recordings for several spike events. d. The root-mean-square (rms) value of the recorded signal (for each point integrated over a timespan of 200ms) is shown for each individual gSGFET as well as the mean value of all devices, revealing a significant improvement of the signal-to-noise ratio.

A reliable comparison of recording quality across different recording sites is difficult in-vivo, as locally recorded signals depend on many factors such as the underlying brain tissue and the adhesion of each 
sensor to it. Therefore, a periodically generated artificial hippocampal population spike (Multi Channel Systems signal generator ME-W-SG) was applied to a phosphate-buffered saline solution (PBS, 10mMol). Figure $4 \mathrm{~b}$ displays one spike event recorded by each transistor of the array. Figure $4 \mathrm{c}$ overlaps all the recordings for several events, demonstrating the improvement of SNR for the UVO-treated devices. To better quantify the SNR, the root-mean-square value of the recording in figure $4 \mathrm{c}$ is plotted in figure $4 \mathrm{~d}$, showing average noise values around $80 \mu \mathrm{V}$ for non-treated and $30 \mu \mathrm{V}$ for UVO-treated devices. This presents an over twofold improvement of signal resolution for such sensor geometry, due to the improved metal-graphene contacts.

\section{Summary}

Here we have shown the critical importance of the contact resistance for the performance of graphene solution-gated field-effect transistors in neural sensing applications. Poor contacts reduce the linearity of the transfer characteristics causing signal distortions, lower the signal-to-noise ratio of the recorded signal and limit the sensor homogeneity. Exposing the contact region of the graphene channel to UV-ozone before deposition of the contacting metal, is shown to significantly improve charge injection at the contacts by a combination of surface cleaning and defect creation. A theoretical model is used to understand the transition from contact to channel dominated noise as a consequence of low contact resistance, and to assess its effect on the total noise of gSGFETs with different channel geometries. Finally, the compatibility of the contact improvement treatment with flexible substrates is validated, demonstrating the fabrication of flexible ECoG-arrays of gSGFETs exhibiting high signal-to-noise ratio.

The development of a novel class of brain-machine interfaces capable of providing further insights into the working paradigms of the brain and granting accurate control of neuro-prostheses might eventually require integrating brain-machine interfaces (BMIs) with single-neuron resolution (cell-body only several $\mu \mathrm{m}$ in size). Considering the increasing dominance of poor contacts on the SNR with decreasing sensor size, improved contact interfaces will become indispensable when moving the gSGFET technology towards this goal. Our work demonstrates a scalable technique to provide high-quality metal-graphene interfaces with low contact resistance, paving the way for low noise, high-density neural interfaces based on graphene transistors.

\section{Acknowledgements}

The authors wish to thank Narcís Mestres-Andreu (ICMAB-Institut de Ciència de Materials de Barcelona) for giving us access to the ultraviolet ozone cleaner. 
This work has received funding from the European Union's Horizon 2020 research and innovation programme under grant agreements No 785219 (Graphene Flagship Core Project 2) and No 732032 (BrainCom). We also acknowledge funding from Generalitat de Catalunya 2017 SGR 1426, and the 2DTecBio (FIS2017-85787-R) funded by Ministry of Science, Innovation and Universities, the State Research Agency (AEI) and the European Regional Development Fund (FEDER/UE). The ICN2 is supported by the Severo Ochoa Centres of Excellence programme, funded by the Spanish Research Agency (AEI, grant no. SEV-2017-0706), and is funded by the CERCA programme / Generalitat de Catalunya. R.G.C. and A.B.C. are supported by the International PhD Programme La Caixa - Severo Ochoa (Programa Internacional de Becas "la Caixa"-Severo Ochoa). EDC acknowledges the Spanish MINECO Juan de la Cierva Fellowship JC-2015-25201.

D.J. and N.M. also acknowledge financial support by Spain's Ministerio de Ciencia, Innovación y Universidades under Grant No. RTI2018-097876-B-C21 (MCIU/AEI/FEDER, UE).

This work has made use of the Spanish ICTS Network MICRONANOFABS partially supported by MICINN and the ICTS 'NANBIOSIS', more specifically by the Micro-NanoTechnology Unit of the CIBER in Bioengineering, Biomaterials and Nanomedicine (CIBER-BBN) at the IMB-CNM.

\section{References}

[1] L. Banszerus et al., "Ultrahigh-mobility graphene devices from chemical vapor deposition on reusable copper," Sci. Adv., vol. 1, no. 6, pp. 1-7, 2015.

[2] J. Conroy et al., "Biocompatibility of Pristine Graphene Monolayers, Nanosheets and Thin Films," no. 1, 2014.

[3] R. R. Nair et al., "Fine structure constant defines visual transparency of graphene," Science (80-. ), vol. 320, no. 5881, p. 1308, 2008.

[4] D. G. Papageorgiou, I. A. Kinloch, and R. J. Young, "Progress in Materials Science Mechanical properties of graphene and graphene-based nanocomposites," Prog. Mater. Sci., vol. 90, pp. 75$127,2017$.

[5] F. Schwierz, "Graphene transistors,” Nat. Publ. Gr., vol. 5, no. May, pp. 4-5, 2010.

[6] F. Bonaccorso, Z. Sun, T. Hasan, and A. C. Ferrari, "Graphene photonics and optoelectronics," Nat. Photonics, vol. 4, no. 9, pp. 611-622, 2010.

[7] C. Bussy, D. Jasim, N. Lozano, D. Terry, and K. Kostarelos, “The current graphene safety landscape-a literature mining exercise,” Nanoscale, vol. 7, no. 15, pp. 6432-6435, 2015. 
[8] T. Kuila, S. Bose, A. K. Mishra, P. Khanra, N. H. Kim, and J. H. Lee, “Chemical functionalization of graphene and its applications," Prog. Mater. Sci., vol. 57, no. 7, pp. 1061$1105,2012$.

[9] K. Kostarelos, M. Vincent, C. Hebert, and J. A. Garrido, "Graphene in the Design and Engineering of Next-Generation Neural Interfaces," Adv. Mater., vol. 29, no. 42, pp. 1-7, 2017.

[10] F. Giubileo and A. Di Bartolomeo, "The role of contact resistance in graphene field-effect devices," Prog. Surf. Sci., vol. 92, no. 3, pp. 143-175, 2017.

[11] Y. Zhang, V. W. Brar, C. Girit, A. Zettl, and M. F. Crommie, "Origin of spatial charge inhomogeneity in graphene," Nat. Phys., vol. 5, no. 10, pp. 722-726, 2009.

[12] A. A. Balandin, "Low-frequency 1/f noise in graphene devices," Nat. Nanotechnol., vol. 8, no. 8, pp. 549-555, 2013.

[13] N. Mavredakis, R. Garcia Cortadella, A. Bonaccini Calia, J. A. Garrido, and D. Jiménez, "Understanding the bias dependence of low frequency noise in single layer graphene FETs," Nanoscale, vol. 10, no. 31, pp. 14947-14956, 2018.

[14] P. Karnatak, T. P. Sai, S. Goswami, S. Ghatak, and S. Kaushal, "Current crowding mediated large contact noise in graphene field-effect transistors," Nat. Commun., vol. 7, pp. 1-8, 2016.

[15] Z. Cheng, Q. Li, Z. Li, Q. Zhou, and Y. Fang, "Suspended graphene sensors with improved signal and reduced noise," Nano Lett., vol. 10, no. 5, pp. 1864-1868, 2010.

[16] S. M. Song, J. K. Park, O. J. Sul, and B. J. Cho, "Determination of work function of graphene under a metal electrode and its role in contact resistance," Nano Lett., vol. 12, no. 8, pp. 3887$3892,2012$.

[17] A. Allain, J. Kang, K. Banerjee, and A. Kis, "Electrical contacts to two-dimensional semiconductors," Nat. Mater., vol. 14, no. 12, pp. 1195-1205, Nov. 2015.

[18] “Low contact resistance metals for graphene based devices," Diam. Relat. Mater., vol. 24, pp. 171-174, Apr. 2012.

[19] W. S. Leong, C. T. Nai, and J. T. L. Thong, "What Does Annealing Do to Metal-Graphene Contacts?," Nano Lett., vol. 14, no. 7, pp. 3840-3847, Jul. 2014.

[20] A. Gahoi, V. Passi, S. Kataria, S. Wagner, A. Bablich, and M. C. Lemme, "Systematic comparison of metal contacts on CVD graphene," in 2015 45th European Solid State Device Research Conference (ESSDERC), 2015, pp. 184-187.

[21] W. S. Leong, H. Gong, and J. T. L. Thong, "Low-Contact-Resistance Graphene Devices with Nickel-Etched-Graphene Contacts,” ACS Nano, vol. 8, no. 1, pp. 994-1001, Jan. 2014. 
[22] H. Y. Park et al., "Extremely Low Contact Resistance on Graphene through n-Type Doping and Edge Contact Design,” Adv. Mater., vol. 28, no. 5, pp. 864-870, 2016.

[23] J. T. Smith, A. D. Franklin, D. B. Farmer, and C. D. Dimitrakopoulos, "Reducing Contact Resistance in Graphene Devices through Contact Area Patterning," ACS Nano, vol. 7, no. 4, pp. 3661-3667, Apr. 2013.

[24] X. Yan et al., "Edge-Contact Formed by Oxygen Plasma and Rapid Thermal Annealing to Improve Metal-Graphene Contact Resistance,” ECS J. Solid State Sci. Technol., vol. 7, no. 2, pp. M11-M15, 2018.

[25] Y. Liang et al., "Ultraviolet/ozone treatLi, Weiment to reduce metal-graphene contact resistance," Appl. Phys. Lett., vol. 102, no. 18, 2013.

[26] G. Liu, S. Rumyantsev, M. Shur, and A. A. Balandin, "Graphene thickness-graded transistors with reduced electronic noise,” Appl. Phys. Lett., vol. 100, no. 3, 2012.

[27] W. Li et al., "Highly reproducible and reliable metal/graphene contact by ultraviolet-ozone treatment," J. Appl. Phys., vol. 115, no. 11, 2014.

[28] G. Liu, S. Rumyantsev, M. Shur, and A. A. Balandin, "Graphene Thickness-Graded Transistors with Reduced Low-Frequency 1/f Noise."

[29] G. Liu, S. Rumyantsev, M. S. Shur, and A. A. Balandin, "Origin of 1/f noise in graphene multilayers: Surface vs. volume,” Appl. Phys. Lett., vol. 102, no. 9, 2013.

[30] G. Xu et al., "Effect of spatial charge inhomogeneity on 1/f noise behavior in graphene," Nano Lett., vol. 10, no. 9, pp. 3312-3317, 2010.

[31] M. L. Noise and G. Field-e, "Mobility-Dependent Low-Frequency Noise in Graphene Field-E ff ect Transistors," no. 10, pp. 8124-8130, 2011.

[32] A. K. M. Newaz, Y. S. Puzyrev, B. Wang, S. T. Pantelides, and K. I. Bolotin, "Probing charge scattering mechanisms in suspended graphene by varying its dielectric environment," Nat. Commun., vol. 3, pp. 734-736, 2012.

[33] S. Chatoor et al., "Charge Noise in Graphene Transistors," Nano Lett., vol. 10, no. 5, pp. 15631567, 2010.

[34] C. Hébert et al., "Flexible Graphene Solution-Gated Field-Effect Transistors: Efficient Transducers for Micro-Electrocorticography,” Adv. Funct. Mater., vol. 28, no. 12, pp. 1-15, 2018.

[35] E. Masvidal-Codina et al., "High-resolution mapping of infraslow cortical brain activity enabled by graphene microtransistors,” Nat. Mater., vol. 18, no. 3, pp. 280-288, 2019.

[36] Y. Matsuda, W. Q. Deng, and W. A. Goddard, "Contact resistance for 'end-contacted' metal- 
graphene and metal-nanotube interfaces from quantum mechanics," J. Phys. Chem. C, vol. 114, no. 41, pp. 17845-17850, 2010.

[37] W. S. Leong, H. Gong, and J. T. L. Thong, "Low-Contact-Resistance Graphene," ACS Nano, 2014.

[38] H. Yu, A. Kutana, and B. I. Yakobson, "Carrier Delocalization in Two-Dimensional Coplanar p-n Junctions of Graphene and Metal Dichalcogenides," Nano Lett., vol. 16, no. 8, pp. 5032-5036, 2016.

[39] P. Zhang, Y. Y. Lau, and R. M. Gilgenbach, "Analysis of current crowding in thin film contacts from exact field solution,” J. Phys. D. Appl. Phys., vol. 48, no. 47, p. 475501, Dec. 2015.

[40] D. C. Kim, D. Y. Jeon, H. J. Chung, Y. Woo, J. K. Shin, and S. Seo, "The structural and electrical evolution of graphene by oxygen plasma-induced disorder," Nanotechnology, vol. 20, no. 37, 2009. 